\title{
Early maternal hypothyroxinemia alters histogenesis and cerebral cortex cytoarchitecture of the progeny
}

\author{
Rosalía Lavado-Autric, ${ }^{1}$ Eva Ausó, ${ }^{2}$ José Victor García-Velasco, ${ }^{2}$ María del Carmen Arufe, ${ }^{1}$ \\ Francisco Escobar del Rey, ${ }^{1}$ Pere Berbel, ${ }^{2}$ and Gabriella Morreale de Escobar ${ }^{1}$
}

\author{
${ }^{1}$ Instituto de Investigaciones Biomédicas Alberto Sols, Consejo Superior de Investigaciones Científicas and \\ Universidad Autónoma de Madrid, Madrid, Spain \\ ${ }^{2}$ Instituto de Neurociencias de Alicante, Universidad Miguel Hernández-Consejo Superior de \\ Investigaciones Científicas, San Juan (Alicante), Spain
}

\begin{abstract}
Epidemiological studies from both iodine-sufficient and -deficient human populations strongly suggest that early maternal hypothyroxinemia (i.e., low circulating free thyroxine before onset of fetal thyroid function at midgestation) increases the risk of neurodevelopmental deficits of the fetus, whether or not the mother is clinically hypothyroid. Rat dams on a low iodine intake are hypothyroxinemic without being clinically hypothyroid because, as occurs in pregnant women, their circulating 3,5,3'triiodothyronine level is usually normal. We studied cell migration and cytoarchitecture in the somatosensory cortex and hippocampus of the 40-day-old progeny of the iodine-deficient dams and found a significant proportion of cells at locations that were aberrant or inappropriate with respect to their birth date. Most of these cells were neurons, as assessed by single- and double-label immunostaining. The cytoarchitecture of the somatosensory cortex and hippocampus was also affected, layering was blurred, and, in the cortex, normal barrels were not formed. We believe that this is the first direct evidence of an alteration in fetal brain histogenesis and cytoarchitecture that could only be related to early maternal hypothyroxinemia. This condition may be 150-200 times more common than congenital hypothyroidism and ought to be prevented both by mass screening of free thyroxine in early pregnancy and by early iodine supplementation to avoid iodine deficiency, however mild.
\end{abstract}

J. Clin. Invest. 111:1073-1082 (2003). doi:10.1172/JCI200316262.

\section{Introduction}

The importance of thyroid hormone transfer from the mother to the fetus during the second half of human pregnancy has received increasing acceptance (1). There is also increasing awareness of the importance of maternal thyroxine for the development of the brain early in pregnancy - that is, before onset at midgestation of its significant secretion by the fetal thyroid, a period during which the mother is the only source of thyroid hor-

Received for publication June 24, 2002, and accepted in revised form January 21, 2003

Address correspondence to: Gabriella Morreale de Escobar, Instituto de Investigaciones Biomédicas Alberto Sols, Consejo Superior de Investigaciones Científicas and Universidad Autónoma de Madrid, Arturo Duperier 4, 28029 Madrid, Spain. Phone: 34-91-397-54-00; Fax: 34-91-585-45-87;

E-mail: gmorreale@iib.uam.es.

Rosalía Lavado-Autric and Eva Ausó contributed equally to this work.

Conflict of interest: The authors have declared that no conflict of interest exists.

Nonstandard abbreviations used: iodine deficiency (ID); congenital hypothyroidism $(\mathrm{CH})$; thyroid-stimulating hormone (TSH); free T4 (FT4); 3,5,3'-triiodothyronine (T3); 2-mercapto-1methylimidazole (methimazole); low-iodine diet (LID); primary somatosensory cortex (S1); 3,3'-diaminobenzidine (DAB); neuronal nuclei (NeuN); 2', $3^{\prime}$-cyclic nucleotide $3^{\prime}$-phosphodiesterase (CNP); posteromedial barrel subfield (PMBSF); type II 5'-iodothyronine deiodinase (5'D-II). mone for the fetus. The experimental evidence and epidemiological findings have recently been reviewed (2-4). Although much of the evidence from human studies is related to maternal hypothyroxinemia caused by iodine deficiency (ID), this conclusion has been extended to pregnant women in the Netherlands (5) and the United States $(6,7)$. Even in the Western populations, children with some degree of neurodevelopmental impairment caused by early maternal hypothyroxinemia may be 150-200 times more common than those with congenital hypothyroidism (CH) (2).

The World Health Organization declared that ID is, after starvation, the single most important cause of preventable brain damage, including different degrees of mental retardation and disabling cerebral palsy. The severity and potential irreversibility of the brain damage are not only related to the degree of ID but also to the period in life during which the individual was exposed to it $(8,9)$. The most marked CNS damage is that of some people with neurological cretinism, who are born in areas of severe ID. The neurological abnormalities include, among others, hearing and speech defects, mental deficiency, and motor defects $(10,11)$, which are clearly related to the mother's ID and her consequent inability to increase circulating thyroxine during pregnancy $(9,12-15)$. Moreover, it has more recently become evident that maternal hypothyroxinemia not only 
results in the birth of children with neurological cretinism but also in decreased mental and psychomotor development of the rest of the population without cretinism (16-19). People with neurological cretinism are only born in areas of very severe ID, but the mental development of the population as a whole is also affected by mild and moderate maternal ID $(19,20)$. The prevention of both the marked CNS damage seen in the people with neurological cretinism and of the mental retardation of the population requires correction of the maternal hypothyroxinemia before midgestation (16, 19-22) or the end of the second trimester (23), before onset of significant fetal thyroid function. Third trimester or early postnatal correction of the low circulating $\mathrm{T} 4$, a measure that is quite effective in preventing most CNS damage in $\mathrm{CH}$, does not reverse the deficits caused by maternal hypothyroxinemia because most of the consequences have become permanent by the end of the second trimester.

The term "hypothyroxinemia" is used here and elsewhere $(2,3)$ to indicate that, whether or not clinical or subclinical (with thyroid-stimulating hormone [TSH] above normal values) hypothyroidism is present, $\mathrm{T} 4$ or free T4 (FT4) is low as compared with values usually found at the same stage of pregnancy in normal women with adequate iodine intake. In the present context, it is important to realize that iodine-deficient women are hypothyroxinemic, but they are not clinically hypothyroid $(9,12)$, because their circulating $3,5,3^{\prime}$-triiodothyronine (T3) is normal or even slightly elevated and sufficient for euthyroidism of most tissues and organs. TSH rarely increases above normal in these women $(24$, 25). Indeed, the severity of the CNS damage and mental retardation of their progeny is causally correlated with the degree of the maternal hypothyroxinemia and not to changes in $\mathrm{T} 3$ or TSH $(17,22)$. In the study by Pop et al. (5) performed in the Netherlands, a country considered to be free of ID, the developmental index of the child was correlated to the first trimester FT4 values of the mother and not to the TSH levels. One out of every two women with first trimester FT4 values below the tenth percentile had a child with a developmental index more than one SD below the mean. None of these women were clinically or subclinically hypothyroid.

Because of obvious ethical strictures, direct evidence of a causal relationship between early maternal hypothyroxinemia and mental development can only be obtained in animal models, such as those previously used to study the effects of maternal ID on the hippocampus of rat fetuses (26). At 21 days of gestational age (E21), there is a delay in the development of radial glial cells of the hippocampus that are involved in neuronal migration. Since fetal thyroid hormone secretion in rats starts at E17.5-E18, the delay could have been caused by the inadequate synthesis of thyroid hormone by the fetal gland. Results neither confirmed nor excluded a role of the maternal hypothyroxinemia before E17.5-E18 - namely, during an earlier period of development equivalent to the first half of human pregnancy.
Using BrdU immunocytochemistry, Lucio et al. (27) and Berbel et al. (28) have shown that neocortical cell migration is defective in the progeny of severely hypothyroid dams that were treated with the goitrogen 2-mercapto-1-methylimidazole (methimazole) from E13 onward. Some of their findings strongly suggested that early maternal thyroid hormone deficiency deranges the migratory pattern of cells into the cortex, either directly by decreasing availability of the hormones to the developing brain or indirectly through poor placental function caused by maternal hypothyroidism. Therefore, it appeared especially interesting to investigate whether such early migratory defects are also found in the progeny of hypothyroxinemic dams that, in contrast to those receiving methimazole, are not "clinically" hypothyroid.

\section{Methods}

Animals and treatments. Wistar rats were housed in temperature-controlled $\left(22-24^{\circ} \mathrm{C}\right)$ animal quarters, with automatic light/dark cycles of $14 / 10$ hours, under veterinary control following European Community guidelines and approval by the ethics committee of our institutions.

Young adult female rats weighing approximately 200 g were fed a diet with a low-iodine content (LID) for 10 days and given $1 \% \mathrm{KClO}_{4}$ as drinking water to lower the initial content of iodine-containing compounds in the thyroid gland, after which the $1 \% \mathrm{KClO}_{4}$ was withdrawn. The rats were divided into three groups. The LID-plus-KI group received LID containing KI to ensure a normal iodine intake (approximately $10 \mu \mathrm{g}$ of iodine per day); the LID-1 group was fed LID alone; and the LID- 2 group was fed LID containing $0.005 \% \mathrm{KClO}_{4}$, added to further decrease thyroid uptake of the small amounts of iodine contained in the LID itself and in the supplements added throughout pregnancy and lactation to prevent nutritional deficiencies other than ID. The LID and the supplements were prepared as described (26). A fourth (normal) group was included that was fed a stock diet for rats (Sandermus from Sanders, Barcelona, Spain) to asses that LID-plus-KI dams were not nutritionally deficient.

After 3 months, blood (approximately $0.5 \mathrm{ml}$ ) was obtained under slight ether anesthesia from the jugular vein to determine $\mathrm{T} 4$ and $\mathrm{T} 3$. The plasma was spun off and kept at $-20^{\circ} \mathrm{C}$. The rats were mated with normal males. Vaginal smears and microscopic visualization of spermatozoa confirmed the day of mating (E0). Each group of animals was subdivided into two subgroups depending on when they were injected intraperitoneally for 3 days with BrdU $(20 \mathrm{mg} / \mathrm{kg}$ per day in physiological saline; Boehringer Mannheim España, Barcelona, Spain), either at E14, E15, and E16 (E14-E16 subgroup) or at E17, E18, and E19 (E17-E19 subgroup).

Four dams from each group were killed at E21 and the weight and number of viable fetuses was recorded. The pups born to the other dams from each group were equaled to eight pups per dam at 2-3 days of postnatal age (P2-P3, with P0 being the day of birth). The different 
feeding regimens were continued after weaning, and at P40 the pups were weighed, anesthetized, bled, and perfused with $50 \mathrm{ml}$ of saline followed by $200 \mathrm{ml}$ of $4 \%$ paraformaldehyde and $0.1 \mathrm{M}$ sucrose in $10 \mathrm{mM} \mathrm{PB}(\mathrm{pH}$ 7.3-7.4) containing $0.002 \% \mathrm{CaCl}_{2}$. Brains were carefully dissected out and postfixed by immersion in perfusion solution at room temperature for 4 hours and then stored at $4^{\circ} \mathrm{C}$ in $\mathrm{PB}$ containing $0.1 \mathrm{M}$ sucrose.

Immunohistochemistry. Parallel series of coronal sections of $100 \mu \mathrm{m}$, cut with a Vibratome and containing the primary somatosensory cortex ( $\mathrm{S} 1$, taken at -2.0 to $-4.0 \mathrm{~mm}$ from bregma) and the mediocaudal portion of the hippocampal formation (at -3.8 to $-4.3 \mathrm{~mm}$ from bregma), were collected in PBS. One series was processed for BrdU immunocytochemistry, and an adjacent series was stained with cresyl violet (SigmaAldrich, St. Louis, Missouri, USA). For BrdU immunocytochemistry, we followed the procedure of Lucio et al. (27), using BrdU mouse Ab (Amersham Pharmacia Biotech Ltd., Little Chalfont, Buckinghamshire, UK), biotinylated horse anti-mouse $\mathrm{Ab}$, an $\mathrm{ABC}$ kit (both from Vector, Burlingame, California, USA), and 3,3'-diaminobenzidine (DAB, Sigma-Aldrich). A second series was incubated only with anti-mature neurons neuronal nuclei (NeuN) mouse $\mathrm{Ab}$ (1:100, Chemicon International Inc., Temecula, California, USA), biotinylated horse antimouse $\mathrm{Ab}, \mathrm{ABC}$, and $\mathrm{DAB}$. $\mathrm{A}$ third series was double immunostained, beginning with incubation with mouse anti-BrdU $\mathrm{Ab}$ and biotinylated horse antimouse $\mathrm{Ab}, \mathrm{ABC}$, and $\mathrm{DAB}$ intensified with $0.4 \%$ ammonium nickel sulfate (29) and continued with incubation with a marker for oligodendrocytes, using anti-2', $3^{\prime}$-cyclic nucleotide $3^{\prime}$ phosphodiesterase (CNP) mouse $\mathrm{Ab}$ (1:100, Chemicon) followed by biotinylated horse anti-mouse $\mathrm{Ab}, \mathrm{ABC}$, and $\mathrm{DAB}$.

Series from the eight subgroups were processed in parallel. Immunostained sections were mounted on gelatinized slides, air dried for 24 hours, dehydrated in ethanol, cleared in xylol, and coverslipped.

Quantification of BrdU-labeled cells. We plotted only BrdUlabeled cells that showed intensely and homogeneously labeled nuclei or diffusely labeled nuclei of lesser intensity but with clumped chromatin. These cells have entered the last $S$ phase of their cell cycle during uptake of the injected BrdU and correspond to types 1 and 2, respectively, of Takahashi et al. (30).

For quantitative analysis, we studied five LID-1 pups (three of the E14-E16 and two of the E17-E19 subgroups), six LID-2 pups (three of the E14-E16 and three of the E17-E19 subgroups), seven LID-plus-KI pups (four of the E14-E16 and three of the E17-E19 subgroups), and six normal pups (three of the E14-E16 and three of the E17-E19 subgroups). Pups were taken from two to three litters per subgroup, with the exception of a single litter for the E17-E19 LID-1 dams. Both in S1 and in hippocampus, BrdU-labeled cells were counted in a total of 9-12 probes per experimental subgroup; the mean values corresponding to each pup were later used for statistical evaluation of the results. In $\mathrm{S} 1$, probes represented by templates generated by the computer system (500-600 $\mu \mathrm{m}$ wide; mean, $550 \mu \mathrm{m}$ wide) (Figure 1g) were placed over the posteromedial barrel subfield (PMBSF or barrel cortex) and spanned from layer I to the subcortical white

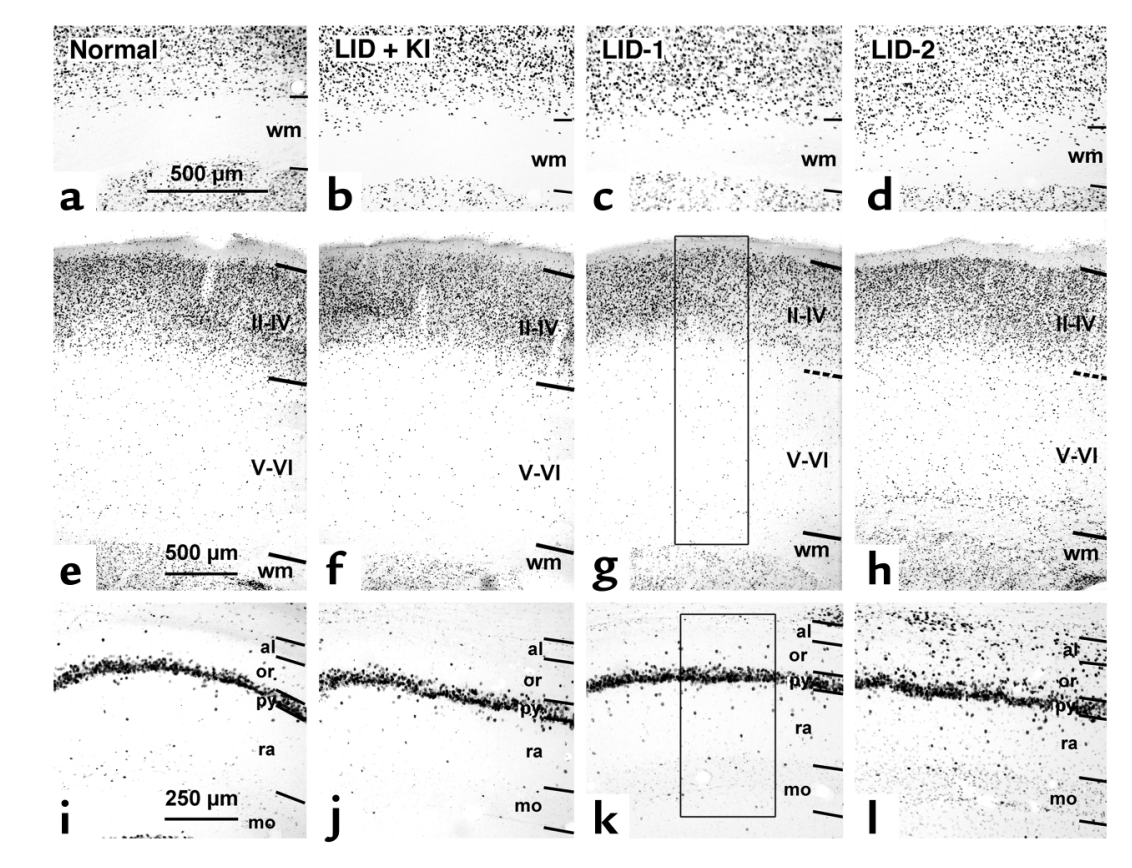

Figure 1

Photomicrographs of coronal sections of primary somatosensory cortex (a-h) and hippocampus (i-I) showing BrdU-labeled cells in normal, LID-plus-KI, LID-1, and LID-2 pups at P40. BrdU labeling after E14-E16 (a-d) and E17-E19 (e-I) injections shows that both in the neocortex $(\mathbf{a}-\mathbf{h})$ and in the hippocampus (i-I), the radial distribution of BrdU-labeled cells is more widespread in LID-1 and LID-2 pups than in normal and LID-plus-KI pups. Note the increased number of heterotopic BrdU-labeled cells in the white matter of the somatosensory cortex ( $\mathbf{c}$ and $\mathbf{d}$ ) and in the strata oriens and alveus of CA1 of the progeny of LID-1 and LID-2 dams ( $\mathbf{k}$ and $\mathbf{I}$ ) as compared with the progeny of normal and LID-plus-KI dams ( $\mathbf{a}$ and $\mathbf{b}$ and $\mathbf{i}$ and $\mathbf{j}$, respectively). Horizontal lines indicate the borders between layers. Dashed lines indicate borders in $\mathbf{g}$ and $\mathbf{h}$, since they are blurred in cresyl violet-stained adjacent sections. Rectangles in $\mathbf{g}$ and $\mathbf{k}$ show two examples of probes. In each probe, BrdU-labeled cells were plotted, and the relative frequency per layer was calculated. Borders between layers were established by superposing adjacent cresyl violet-stained sections. Strata pyramidale, radiatum, and moleculare are indicated. Magnification for $\mathbf{a}-\mathbf{d}$ is $30 \times$; $17 \times$ for $\mathbf{e}-\mathbf{h}$; and $36 \times$ for i-I. wm, white matter; or, stratum oriens; al, stratum alveus; py, stratum pyramidale; ra, stratum radiatum; mo, stratum moleculare; II-IV, layers II through IV; V-VI, layers V through VI. 
Table 1

Circulating thyroid hormones in dams from the experimental groups, their reproductive performance, and the body weights of their P40 progeny

\begin{tabular}{|c|c|c|c|c|c|c|}
\hline & $\begin{array}{c}\text { Serum T4 } \\
(\mathrm{ng} / \mathrm{ml})\end{array}$ & $\begin{array}{c}\text { Serum T3 } \\
(\mathrm{ng} / \mathrm{ml})\end{array}$ & $\begin{array}{c}\text { Number of fetuses } \\
\text { per litter }\end{array}$ & $\begin{array}{l}\text { Fetal weight } \\
(\mathrm{g})\end{array}$ & $\begin{array}{l}\text { Weight of litter } \\
(\mathrm{g})\end{array}$ & $\begin{array}{l}\text { Weight at P40 } \\
(\mathrm{g})\end{array}$ \\
\hline Normal & $35.9 \pm 6.3$ & $0.73 \pm 0.02$ & $11.0 \pm 0.9$ & $5.2 \pm 0.1$ & $51.5 \pm 2.3$ & $131 \pm 4$ \\
\hline LID-plus-KI & $26.1 \pm 2.7$ & $0.69 \pm 0.06$ & $10.0 \pm 1.4$ & $5.1 \pm 0.1$ & $50.9 \pm 6.3$ & $132 \pm 4$ \\
\hline LID-1 & $<2.5^{\mathrm{A}}$ & $0.65 \pm 0.15$ & $9.8 \pm 0.9$ & $5.2 \pm 0.1$ & $50.6 \pm 5.3$ & $130 \pm 3$ \\
\hline LID-2 & $<2.5^{\mathrm{A}}$ & $0.37 \pm 0.06^{\mathrm{A}}$ & $9.8 \pm 1.6$ & $5.2 \pm 0.0$ & $46.6 \pm 2.3$ & $129 \pm 4$ \\
\hline
\end{tabular}

Results are shown as mean values \pm SEM. ${ }^{A}$ The difference was statistically significant as compared with the LID-plus-KI group.

matter. In the hippocampus, probes $(300-400 \mu \mathrm{m}$ wide; mean, $350 \mu \mathrm{m}$ wide) (Figure $1 \mathrm{k}$ ) were placed over the CA1 and CA 3 areas and spanned from strata oriens to moleculare (in CA1, they also included the alveus). In all cases, the borders between layers and strata were placed at the same relative depth, as measured from adjacent cresyl violet-stained sections. For each subgroup, the relative frequencies of labeled cells in each layer were averaged across probes and animals.

Determination of serum T3 and T4. Both concentrations were determined by very sensitive and highly specific radioimmunoassays (31). The small amounts of available serum precluded the determination of TSH levels.

Statistical analysis. For body weights, numbers of fetuses per litter, litter weights, and concentrations of circulating iodothyronines, we used one-way ANOVA and the protected least-significant difference test for multiple comparisons, after validation of the homogeneity of variances by the Bartlett-Box $F$ test, using the SPSS statistical package (SPSS Inc., Chicago, Illinois, USA). Plots and counts of BrdU-labeled cells were obtained using the Neurograph system (Microptic, Barcelona, Spain). The Systat statistical software (Systat Inc., Evanston, Illinois, USA) was used for two-way ANOVAs of cell density, the factors being layers in various brain areas and experimental groups. Significant layer-group interactions $(P<0.001)$ were found in the cortex and in hippocampal CA1 and CA3 both for the E14-E16 and E17-E19 subgroups. One-way ANOVAs of cell densities were then used to identify layers affected by the experimental condition, followed by Tukey's test to identify significant differences between means. $P=0.05$ was considered significant in all comparisons.

\section{Results}

Table 1 summarizes the effects of the different diets on $\mathrm{T} 4$ and $\mathrm{T} 3$ just before mating. Results confirm previous ones in LID-1 and LID-2 dams $(26,32-34)$. T4 values in the LID-1 dams were well below normal $(<10 \%$ of the values of LID-plus-KI dams), and T3 values remained normal. In LID-2 dams, however, T3 values decreased, though much less markedly than T4. Despite the decrease in T3 values, the reproductive performance of these animals was normal, as was the postnatal growth of the pups at $\mathrm{P} 40$.

In normal and LID-plus-KI pups, the radial distribution of BrdU-labeled cells in S1 was consistent with that described previously by Bayer and Altman (35), showing the normal "inside-out" gradient model of radial migration. In the E14-E16 subgroups of both LID-1 and LID-2 progeny, BrdU-labeled cells were observed in deep layers, whereas in the E17-E19 subgroups, they were in more superficial layers. These findings indicated that most of the cortical cells of the LID-1 and LID-2 pups also followed a normal "insideout" distribution. Despite this, the distribution of BrdU-labeled nuclei was more widespread in LID-1 and LID-2 pups than in normal and LID-plus-KI progeny, showing an abnormal number of heterotopic BrdUlabeled cells - that is, cells found in layers different from those corresponding to their birth date and at aberrant locations, such as the subcortical white matter (Figure 1, a-d). As a general feature, the percentage of BrdU-labeled cells in LID-1 and LID-2 pups decreases in superficial layers and increases in deeper ones as compared with normal pups (Figure 1, e-h). Thus, in LID-1 and LID-2 progeny, the proportion of BrdUlabeled cells labeled at E14-E16 decreased in layer VI and increased in the subcortical white matter (see $P$ values in Figure 2, a and b), whereas the proportion of BrdU-labeled cells at E17-E19 decreased in layers II-III and IV and increased in layers V and VI and in white matter. In LID-2 pups, heterotopic BrdU-labeled cells in white matter were observed after both E14-E16 and E17-E19 injections $(8.0 \% \pm 1.6 \%$ and $5.3 \% \pm 1.4 \%$, respectively, as compared with $<0.6 \%$ for the normal and LID-plus-KI subgroups). In LID-1 pups, BrdUlabeled cells were mostly observed after E14-E16 injections $(7.2 \% \pm 0.6 \%$ at E14-E16 and $1.4 \% \pm 0.4 \%$ at E17-E19 as compared with $<0.6 \%$ for the normal and LID-plus-KI subgroups).

The radial distributions of BrdU-labeled cells in CA1 and CA3 of both subgroups of normal and LID-plusKI progeny were similar and consistent with previous descriptions for normal rats (36). In contrast, their distribution was more widespread in LID-1 and LID-2 pups, both in CA1 and CA3, showing an abnormal number of heterotopic BrdU-labeled cells (Figure 1, i-1). As a general feature, both at E14-E16 and at E17-E19, the percentage of BrdU-labeled cells in CA1 of LID-1 and LID-2 pups decreases with respect to normal and LID-plus-KI rats in the pyramidal layer (see $P$ values in Figure 2, c-f) and increases in strata alveus and oriens. In CA3 of both subgroups, the percentage of BrdU-labeled cells also decreases with respect to normal and LID-plus-KI rats in the pyramidal layer, but 
increases in the outer plexiform layer. No BrdU-labeled cells were found in the alveus of any of the subgroups of normal and LID-plus-KI pups.

In addition to an aberrant distribution of BrdUlabeled cells, there were changes in the cytoarchitecture of the barrel cortex and of the hippocampus (cresyl violet-stained sections) in LID-1 and LID-2 pups. The barrel cortex of LID-plus-KI pups (Figure 3a) was similar to that of normal pups (not shown), and the major cytoarchitectonic features are typical barrels that can be seen in layer IV, large pyramids in layer V, and easily distinguishable borders within all layers. In contrast, these features are less prominent in the progeny of LID-1 and LID-2 dams (Figure 3, b and c); barrels are not clearly defined in layer IV (neurons were more homogeneously distributed and septae that demarcated barrels were not seen), pyramids in layer $V$ are smaller than normal, and the borders between layers II-VI are blurred. However, no differences were found in the thickness of the cortex among the four groups (on average, $1744 \pm 42 \mu \mathrm{m}$ in normal and LID-plus-KI pups versus $1738 \pm 133$ in LID-1 and LID-2 pups).

In the hippocampus, as in the barrel cortex, the gross cytoarchitecture of LID-plus-KI pups (Figure 3, d-f) was similar to that of normal pups (data not shown). In CA1, the pyramidal cell layer showed clear-cut borders with the adjacent strata. In contrast, both in CA1 and CA3 of LID- 1 and LID- 2 progeny, the border of the pyramidal layer with the strata oriens was more blurred (Figure 3, g-i). In addition, the stratum radiatum in CA1 was $14 \%$ thinner than normal (on average, $268 \pm 17 \mu \mathrm{m}$ in normal and LID-plus-KI pups versus $231 \pm 23 \mu \mathrm{m}$ in LID-1 and LID-2 pups).

NeuN immunostaining confirmed the results found in cresyl violet-stained sections, where the borders between layers were blurred (Figure 4, a and b), and showed an increased number of heterotopically located neurons in the subcortical white matter (Figure 4, $c$ and d) and in hippocampal strata oriens and alveus (Figure 4, e and f) of LID rats, as compared with LID-plus-KI and normal rats. CNP-positive oligodendrocytes showed a light-brown-stained cell body and processes (Figure 4g). The cell body of CNP-positive oligodendrocytes (between $5-8 \mu \mathrm{m}$ in diameter) was smaller than that of nuclei of most of the BrdUlabeled cells (between $10-15 \mu \mathrm{m}$ in diameter). In both the E14-E16 and the E17-E19 subgroups of LID rats and their controls, some cells that were both BrdU-labeled and CNP-positive were occasionally seen, but in very small numbers. These double-labeled cells

Figure 2 showed a partial BrdU labeling that was limited to the clumped chromatin corresponding to the type 3 cells described by Takahashi et al. (30) and were not counted by us. No CNP-positive cytoplasm and processes were seen in close contact with type 1 and 2 BrdU-labeled nuclei.

\section{Discussion}

The experimental design. We have shown previously (26, 32-34) that the present experimental design is quite different from that of models used to induce severe hypothyroidism in dams and/or pups. The latter usually involve surgical thyroidectomy of the dams or their treatment with goitrogens, such as methimazole (26, 31 ). In contrast with thyroidectomized and with goitrogen-treated dams, the present LID-1 and LID-2 dams could not be distinguished from their controls by physical appearance, by growth, or by their reproductive performance. Neither were visible "clinical" signs of neonatal or juvenile hypothyroidism (poor hair, different skull proportions, abnormal locomotion, poor growth) observed in their progeny. In these different experimental models, the concentrations of $\mathrm{T} 4$ and $\mathrm{T} 3$ that we found in embryonic and fetal samples and in placentas before E18 reflect the pattern of changes

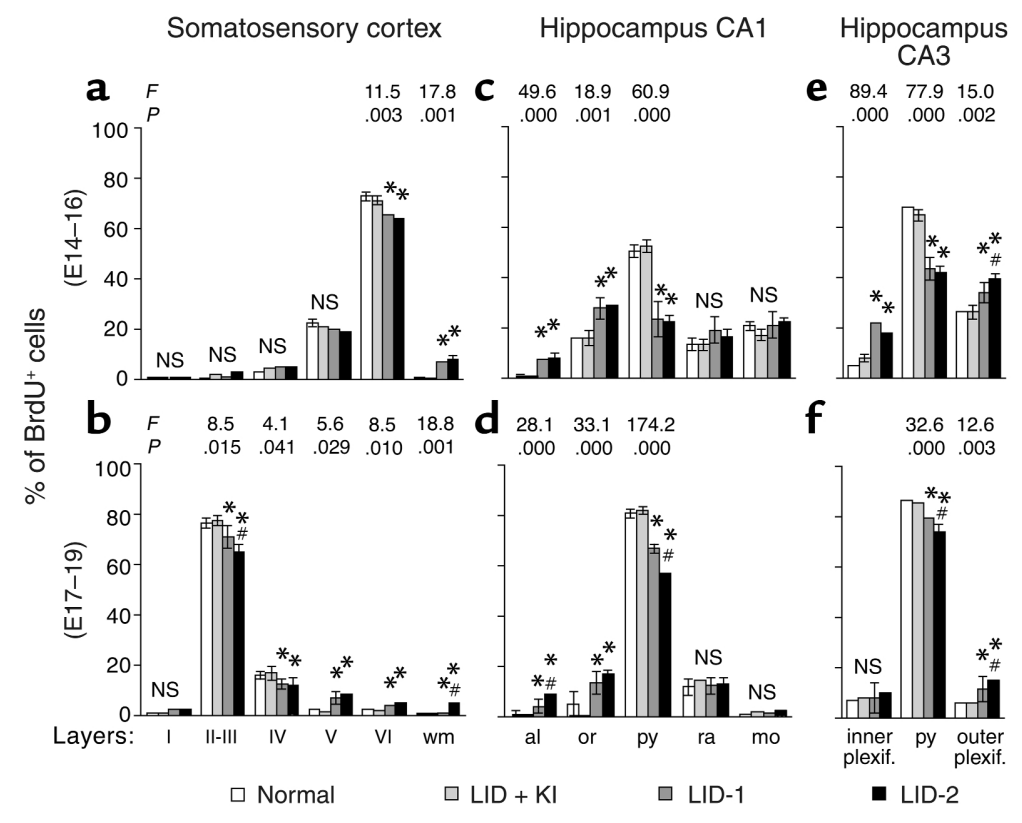

Histograms showing the percentage of BrdU-labeled cells in various layers of the primary somatosensory cortex and the CA 1 and CA 3 regions of the hippocampus. Results for the progeny of the E14-E16 subgroups of normal, LID-plus-KI, LID-1, and LID-2 dams are shown in a, c, and e. Results for the E17-E19 subgroups are shown in $\mathbf{b}, \mathbf{d}$, and $\mathbf{f}$. Error bars represent SD across layers from the same group. Single asterisk indicates a significant difference between the LID-1 or LID-2 group and their control group (LID-plus-KI and normal groups); crosshatch indicates a significant difference between the LID-1 group and the LID-2 group. The $F$ and $P$ values corresponding to layers for which significant differences were found between groups are shown in the upper part of each panel. The degrees of freedom were 3, 9 for the E14-E16 subgroups and 3, 7 for the E17-E19 subgroups. inner plexif., inner plexiform stratum; outer plexif., outer plexiform stratum. 


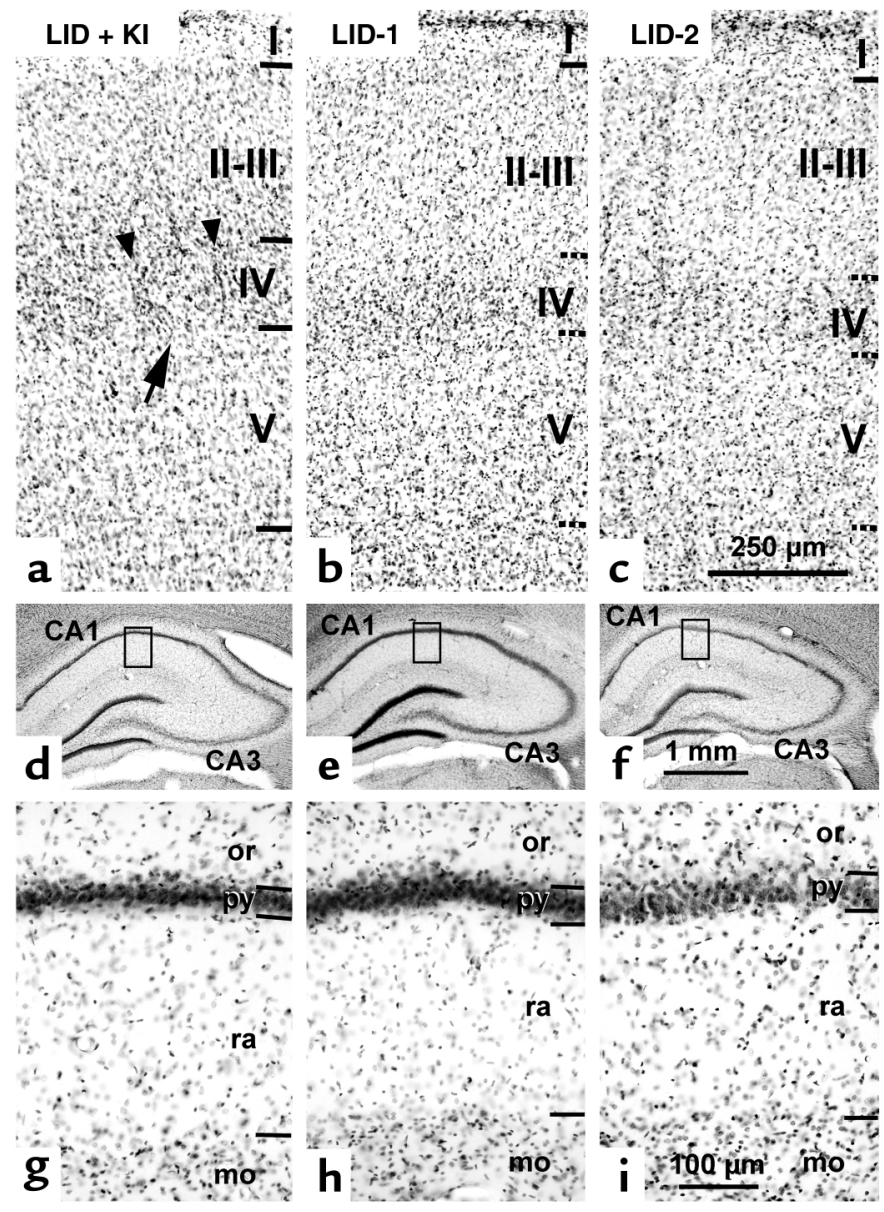

observed in the maternal plasma $(31,37,38)$. Thus, the thyroidectomized dams have very low circulating levels of T4 and T3 up to E21, as do all samples obtained from the conceptus between E9 and E18 - namely, E9-E12 embryotrophoblasts, E9-E12 placentas, and E13-E17 whole embryos. The mean number of fetuses per litter $(6.8 \pm 0.3)$, the mean fetal weight $(4.2 \pm 0.1 \mathrm{~g})$, and the mean weight of the litter $(26.8 \pm 1.0 \mathrm{~g})$ of viable fetuses at E21 were markedly reduced. In contrast, in the hypothyroxinemic LID-1 dams, we have found $(32-34,39)$ that circulating T4 is very low up to E21, as are the $\mathrm{T} 4$ concentrations in $\mathrm{E} 11$ embryotrophoblasts and in E17 placentas and whole embryos. The corresponding T3 concentrations, however, usually remain normal (E11) or decrease (E17 placentas and embryos), but to a much lesser extent than T4. This decrease occurred also in the present LID-2 dams, despite the fact that, as indicated above, neither the dams nor the progeny were "clinically" hypothyroid.

Whether the concentrations of $\mathrm{T} 4$ and $\mathrm{T} 3$ in the brain of LID fetuses before E17-E18 follow a different pattern than suggested by the maternal circulating levels of both iodothyronines has not been clarified, because of the lack of sensitivity of available methods. Information obtained for fetuses of thyroidectomized dams (38) revealed that the activity of type II 5 -iodothyronine

\section{Figure 3}

Photomicrographs of cresyl violet-stained coronal sections showing the cytoarchitecture of the PMBSF of the primary somatosensory cortex $(\mathbf{a}-\mathbf{c})$ and hippocampal CA1 $(\mathbf{d}-\mathbf{i})$ in LID-plus-KI, LID-1, and LID-2 progeny at P40. In the PMBSF of LID-plus-KI pups, borders between layers are clear-cut, as expected in normal animals (horizontal lines in a), whereas in LID-1 and LID-2 pups they are blurred (horizontal dashed lines in $\mathbf{b}$ and $\mathbf{c}$ ). In layer IV of LID-plus-KI pups, barrels are normal and well defined, as indicated by an arrow in $\mathbf{a}$, and demarcated by septae (arrowheads). In contrast, barrels in layer IV of LID-1 and LID-2 pups (b and $\mathbf{c}$ ) are not seen. Enlargements of the insets outlined in $\mathbf{d}-\mathbf{f}$ are shown in $\mathbf{g}-\mathbf{i}$. In the hippocampus, the differences between LID-plus-KI pups and both LID-1 and LID-2 pups are not prominent at low magnification (compare $\mathbf{d}$ with $\mathbf{e}$ and $\mathbf{f}$ ). However, at a higher magnification, the border between strata pyramidale and oriens in CA1 of LIDplus-KI pups is more clear-cut than in LID-1 and LID-2 progeny (compare $\mathbf{g}$ with $\mathbf{h}$ and $\mathbf{i}$ ). Note that strata radiatum is thinner in LID-1 and LID-2 pups than in LID-plus-KI pups. The stratum moleculare is also indicated. Magnification for $\mathbf{a}-\mathbf{c}$ is $54 \times$; $8 \times$ for $\mathbf{d}-\mathbf{f}$; and $76 \times$ for $\mathbf{g}-\mathbf{i}$.

deiodinase $\left(5^{\prime} \mathrm{D}-\mathrm{II}\right)$ in the fetal brain was not influenced by maternal thyroidectomy alone and that both $\mathrm{T} 4$ and $\mathrm{T} 3$ at $\mathrm{E} 17$ were decreased, as expected from the decreased maternal circulating levels. When the thyroidectomized dams were given methimazole and fetal thyroid function was impaired, $5^{\prime} \mathrm{D}$-II activity did not appear to be upregulated by the decrease in fetal T4 until E19 or later $(34,38,39)$. In the fetuses of LID dams, an increase in $5^{\prime} \mathrm{D}$-II activity was also not observed until E19 and was insufficient for the maintenance of normal cerebral T3.

Early effects on the development and organization of S1 and hippocampus. A significant number of heterotopic cells were found that are abnormally located in the S1 and in the hippocampus of the progeny of both LID-1 and LID-2 dams, although neither the pups nor the dams were hypothyroid. NeuN single staining shows that the heterotopic cells that were found in the subcortical white matter of pups from the E14-E16 subgroups of both LID-1 and LID-2 animals are mostly neurons. In addition, BrdU-labeled cells from the E14-E16 and E17-E19 subgroups of LID pups and their controls are not likely to be oligodendrocytes: no type 1 or type 2 BrdU-labeled cells were found that were double-labeled for CNP. In contrast, the number of type 3 BrdU-labeled cells that were also CNP-positive was not negligible, especially in E17-E19 subgroups. These might have been oligodendrocytes that had undergone successive mitoses before P40. Our results, therefore, led us to conclude that maternal hypothyroxinemia clearly results in an alteration of neuronal migration during neocorticogenesis, but the possibility cannot be excluded that the abnormal migration might also affect glial cells, especially those oligodendrocytes that reached the neocortex from the ganglionic eminence through tangential migration $(40,41)$. 
The observed alterations do not correspond merely to a delay of the normal migratory process, because heterotopic cells are found in aberrant destinations (i.e., subcortical white matter and stratum alveus of hippocampal CA1), where they are hardly ever found at any BrdU injection time in the normal animals that we have studied or in the subcortical white matter of normal rats injected with BrdU between E12 and E15 (42). We wish to point out that the aberrant migration of cortical neurons labeled at E14-E16 into the white matter clearly identifies a very important alteration in brain development that is entirely caused by the maternal thyroid hormone deficiency alone. These neurons incorporated $\mathrm{BrdU}$ in the last $\mathrm{S}$ phase of their cell cycle, the latest ones incorporating it at E16, starting migration from the ventricular zone and reaching the cortical plate before E18 (35). If the onset of the migration process in the LID fetuses were delayed for $2-3$ days, it would overlap with that of fetal thyroid function, and the observed abnormalities could no longer be related to the maternal hypothyroxinemia alone. However, the systematic finding of labeled cells in aberrant locations is not explained by such a delay alone. Moreover, when goitrogen-treated dams were given single pulses of BrdU at various times between E14 and P0, no differences were found between the pups from the normal and hypothyroid dams in the proportion of labeled cells in S1, whatever the timing of the BrdU injections (28). After labeling at E19, the number of labeled cells was equally very low in both groups of pups. The findings reported here support the above conclusion that the migratory process we have studied in the E14-E16 subgroups takes place before the onset of fetal thyroid hormone secretion at E17.5-E18 and can, therefore, only be related to alterations of maternal, but not of fetal, thyroid function.

This present conclusion is strongly supported by the preliminary results with a different experimental model (43). Rat dams receiving methimazole for only 3 days (E12, E13, and E14) were subdivided into E14-E16 and E17-E19 subgroups. Abnormalities in cell migration similar to those described here were found in the cortex and hippocampus of the progeny of both subgroups, despite rapid normalization of the maternal thyroid status after withdrawal of methimazole on E15. The observed abnormalities were prevented by the infusion of $\mathrm{T} 4$ into the methimazole-treated dams.

The amount of BrdU incorporated into the cell, the time of injection, and the clearing time of $\mathrm{BrdU}$ (6-8 hours on average) might affect the number of labeled cells. However, the present findings are not likely to be caused merely by small differences between experimental groups in the injection time, considering that different litters were used for each group at each injection period in order to minimize this possibility.

The mechanisms underlying abnormal migration in LID pups are not known and need further study. In this respect, it is interesting that a delayed maturation of the hippocampal radial glia has been found in LID fetuses at E21 (26). This delay in the maturation of radial glia might also occur in the neocortex and might reflect cellular and molecular alterations that would
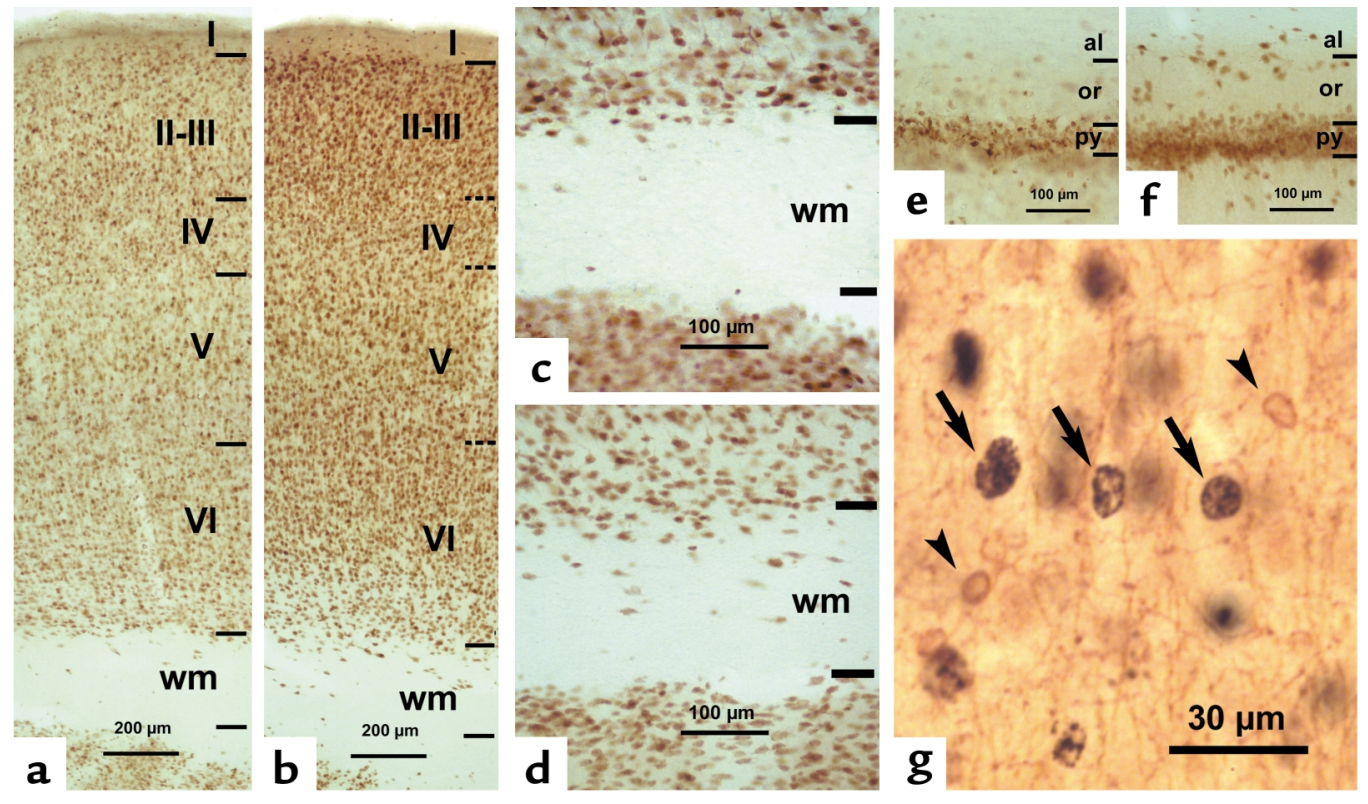

Figure 4

Photomicrographs of NeuN-immunostained coronal sections of the primary somatosensory cortex (a-d) and hippocampal CA1 (e and $\mathbf{f})$ in LID-plus-KI (a, c, and $\mathbf{e}$ ) and LID-1 (b, d, and $\mathbf{f}$ ) progeny at P40. In the neocortex of LID-1 pups, borders between layers are more blurred (horizontal dashed lines in $\mathbf{b}$ ) than in LID-plus-KI rats (horizontal lines in a). The number of NeuN-labeled neurons increases both in subcortical white matter and in strata oriens and alveus of hippocampal CA1 of LID-1 rats ( $\mathbf{d}$ and $\mathbf{f}$, respectively) as compared with LID-plus-KI rats (c and e). In g, CNP-positive oligodendrocytes (arrowheads) and BrdU-labeled nuclei (arrows) are shown in layer V of a LID-1 rat from an E14-E16 subgroup. Note that CNP-positive oligodendrocytes are BrdU negative. 
interfere with normal cell migration at earlier stages of histogenesis, affecting not only their radial but also their tangential migration. In addition to abnormal migration, histogenetic abnormalities might occur in LID rats, affecting neuronal generation and survival. In particular, a survival of some neurons generated at E14-E16 - namely, those transiently destined for the subplate - cannot be excluded. Thus, in LID rats, heterotopic neurons might result not only from an altered migration but also from an abnormal stabilization of neurons that would normally be eliminated. Whichever the case, the migration pattern in fetuses of the LID-1 and LID-2 groups is different from normal.

With experimental models involving prolonged goitrogen treatment of the dams, it is not possible to clarify whether the resulting changes in brain development of their progeny are caused directly by a decreased availability of thyroid hormones to the developing brain or indirectly through hypothyroidism of the placenta, which could affect the entire development of the fetus. This second possibility is quite unlikely in the case of the present LID-1 and LID-2 dams, because although they were severely hypothyroxinemic, their placental function was spared, as shown by their normal reproductive competence. Another epigenetic factor affecting CNS development, such as malnutrition, can also be excluded: results from the LID-plus-KI group could be superimposed on those from the normal animals, confirming that the present supplemented LID was nutritionally adequate (26). The normal litter size, fetal weights, and postnatal growth of the progeny from the LID-1 and LID-2 dams also shows that malnutrition was not a causal factor for the abnormalities in brain development described here.

The finding that the neuronal migration pattern and cytoarchitectonic organization are abnormal in the LID-1 pups, born from mothers with markedly reduced circulating T4 but with normal T3, also supports previous conclusions that availability to the developing brain of maternal T4 is of greater importance than that of T3, because T3 in the fetal brain is almost entirely dependent on its local generation from T4 $(4,44)$ and not on the uptake of circulating T3. The same occurs in human ID, in which the cerebral damage is correlated with the maternal hypothyroxinemia, not with maternal T3 or TSH $(17,22)$. Present results do not exclude that continuing maternal hypothyroxinemia later in pregnancy and/or persisting postnatal hypothyroxinemia of the progeny might result in even more CNS damage and the derangement of a greater spectrum of functional abnormalities (23). It might, for instance, also affect migratory patterns of cells undergoing continuous neurogenesis, such as that occurring in the hippocampus of mammals including rats (45) and humans (46), and the functional properties of newly generated hippocampal cells (47), which might explain CNS deficits not related to early pregnancy.

The abnormalities in neuronal migration and the subtle changes in cytoarchitectonic organization found in the progeny of both LID-1 and LID-2 dams indicate that the normal process of brain maturation and, consequently, the establishment of normal brain functions are likely to be impaired. In this respect, it is interesting that the progeny from LID dams show increased audiogenic seizure susceptibility $(37,48)$. The observed reduction in the thickness of the stratum radiatum in LID-1 and LID-2 pups suggests a reduction in the length of apical dendrites of pyramidal neurons, such as has been reported in granule and pyramidal cells of the hippocampus of goitrogen-treated hypothyroid rat pups (49). It is very likely that abnormal migration also results in aberrant circuits in the neocortex and the hippocampus in the progeny of LID-1 and LID-2 dams, considering that changes in GABAergic (50) and in callosal $(27,51)$ and thalamocortical connections (29) have been found in goitrogen-treated rat pups, in which altered migration also occurs and barrel formation is deranged. Identification of detectable functional deficits caused in the rat by the anatomical alterations described here will require further studies.

Recent studies by others (52) have also focused on the importance of maternal thyroid hormone insufficiency early in pregnancy. Alterations of the expression of several cerebral genes were found in the fetuses of thyroidectomized or goitrogen-treated dams, but they did not persist and returned to normal within a few days; they were also found in rats that started receiving goitrogens as adults. The significance of these observations for brain development are not well understood, since the reported genes are not known to be clearly involved in any developmental processes.

The present study is, to our knowledge, the first to show an effect of early maternal hypothyroxinemia on the histogenesis and cytoarchitecture of the cerebral cortex of the fetus, whether or not circulating $\mathrm{T} 3$ decreases, and without clinical manifestations of hypothyroidism. Although we have not performed direct experiments to define the developmental period during which these effects might be avoided by correction of the maternal hypothyroxinemia with iodine or $\mathrm{T} 4$, it appears reasonable to assume that the alterations would soon become irreversible. To our knowledge, cells that are found in aberrant locations do not later reach their normal destinations. The intrinsic and extrinsic factors (i.e., radial glial guidance, extra- and intracellular signaling, etc.) that allow cortical neuroblasts to complete their process of migration change very rapidly. In particular, radial glia differentiate to cortical astrocytes, extracellular signaling molecules are expressed transiently, and the complexity of neuropile increases as neuroblasts differentiate and the process of synaptogenesis begins around birth in the rat and by the twenty-fourth week of gestation in humans (53). All these changes associated with cortical development and maturation begin after neurogenesis and strongly suggest that cells trapped in aberrant locations could not later reach their normal destinations, even if an appropriate treatment with iodine or $\mathrm{T} 4$ were then 
instituted. The findings summarized above from the experiment in which dams were treated with methimazole for only 3 days also support this conclusion (38). Possible implications for maternal bypothyroxinemia in bumans. Present results may help our understanding of epidemiological findings in man. In humans, heterotopic cells have been described in the neocortex of therapeutically aborted fetuses from an ID region (54). These heterotopic cells can also be observed in human neurological disorders, such as epilepsy (55, 56). There are important differences in the timing of neocorticogenesis between humans and rats with respect to the stages of pregnancy. In humans, neocortical development occurs between the sixth and twenty-fourth week of gestation, with the cortical plate beginning to form by E54 (by the eighth week of gestation). The bulk of cortical cell migration occurs between the eighth and twenty-fourth week of gestation - namely, before the end of the second trimester (53) and mostly before onset of fetal thyroid hormone secretion, at midgestation. In the rat, neocorticogenesis begins comparatively later (by E13) and is not over until a few days after birth, with the major part of the process occurring between E14 and E19, nearer to birth than in humans but again before onset of fetal thyroid function (at E17.5-E18). Thus, despite the differences in timing of neurodevelopmental events between both species when birth is taken into account, similarities may be established when onset of fetal thyroid secretion is considered.

Two main waves of cell migration occur in the human neocortex that take place during the first half of gestation, with peaks at 11 and 14 weeks of gestational, or postmenstrual, age (equivalent to 9 and 12 weeks of postconceptional, or fetal, age, respectively) (57), roughly corresponding to the fetal ages studied here in rats. Our results in rats therefore suggest that these migrations could also be deranged in the progeny of mothers who have been hypothyroxinemic during the first half of pregnancy, a possibility that we cannot ethically confirm or disprove. The subtle changes in migration and cytoarchitecture described here may well be the underlying cause of the decreased mental development described in children born in areas of mild and moderate ID (19), whose mothers are hypothyroxinemic without being hypothyroid. They may also be related to the mental deficits of children born from mothers whose hypothyroxinemia early in pregnancy might be due to other causes $(2,4,5,7)$. Although the mental impairment attributed to early maternal hypothyroxinemia is usually not as severe as that of children with $\mathrm{CH}$ who are deprived of early thyroid hormone treatment, the number of children at risk for neurodevelopmental deficits related to early maternal hypothyroxinemia is $150-200$ times greater than that of newborns with $\mathrm{CH}$.

Maternal hypothyroxinemia is not detected in most pregnant women early in pregnancy, because it does not necessarily result in clinical or even "subclinical" hypothyroidism. The mother herself may synthesize and secrete enough T4 and T3 to meet her own needs, but the amount of $\mathrm{T} 4$ reaching the fetus might not be sufficient for its normal neurodevelopment. The most frequent cause of maternal hypothyroxinemia worldwide is ID, even if mild or moderate; it is not only still common among European pregnant women (25) but is also beginning to be of concern in the United States (58).

However, ID is not necessarily the only cause of maternal hypothyroxinemia occurring without an increase in TSH above normal values. The present results strongly support our previous conclusion (2) regarding the need to establish mass screening programs of pregnant women early in gestation. For the sake of the unborn child, these programs should be based primarily on first trimester parameters related to circulating $\mathrm{T} 4$ and not only on the detection of thyroid antibody positivity and increased serum $\mathrm{TSH}$, as previously proposed for the sake of the mother (59).

In conclusion, present results suggest that, during development, thyroid hormones have selective effects on processes that take place early in fetal development, such as migration during histogenesis and the cytoarchitectural organization of the cerebral cortex. Any situation resulting in a decreased availability of $\mathrm{T} 4$ to the fetal brain is potentially adverse for neurodevelopment. Maternal hypothyroxinemia appears to be a much more frequent cause of deficits in the progeny than $\mathrm{CH}$, for which we have successful neonatal thyroid screening programs.

\section{Acknowledgments}

This study is dedicated to the memory of Andries Querido, whose pioneering studies during the sixties in Papua New Guinea drew attention to the crucial role of maternal thyroid function in the etiology of CNS damage caused by iodine deficiency. This work was financed by Spanish grants CICYT SAF2000-0152C02-01 (to P. Berbel) and CAM 08.5/0059.1/2000 (to G. Morreale de Escobar).

1. Burrow, G.N., Fisher, D.A., and Larsen, P.R. 1994. Maternal and fetal thyroid function. N. Engl. J. Med. 331:1072-1078.

2. Morreale de Escobar, G., Obregón, M.J., and Escobar del Rey, F. 2000. Is neuropsychological development related to maternal hypothyroidism, or to maternal hypothyroxinemia? J. Clin. Endocrinol. Metab. 85:3975-3987.

3. Glinoer, D., and Delange, F. 2000. The potential repercussions of maternal, fetal, and neonatal hypothyroxinemia on the progeny. Thyroid. 10:871-877.

4. Calvo, R.M., et al. 2002. Fetal tissues are exposed to biologically relevant free thyroxine concentrations during early phases of development. J. Clin. Endocrinol. Metab. 87:1768-1777.

5. Pop, V., et al. 1999. Low normal maternal free T4 concentrations during early pregnancy are associated with impaired psychomotor development in infancy. Clin. Endocrinol. (Oxf.) 50:149-155.

6. Man, E.B., and Serunian, S.A. 1976. Thyroid function in human pregnancy. Development or retardation of 7-year-old progeny of hypothyroxinemic women. Am. J. Obstet. Gynecol. 125:949-957.

7. Haddow, J.E., et al. 1999. Maternal thyroid deficiency during pregnancy and subsequent neuropsychological development of the child. N. Engl.J. Med. 341:549-555.

8. Hetzel, B.S. 1983. Iodine deficiency disorders (IDD) and their eradication. Lancet. 2:1126-1129.

9. Hetzel, B.S. 1994. Historical development of concepts of brain-thyroid relationships. In The Damaged brain of iodine deficiency. J.B. Stanbury, editor. Cognizant Communication Co. Elmsford, New York, USA. 1-8. 
10. DeLong, G.R. 1989. Observations on the neurology of endemic cretinism. In Iodine and the brain. G.R. DeLong, J. Robbins, and P.G. Condliffe, editors. Plenum Press. New York, New York, USA. 231-238.

11. Halpern, J.P., et al. 1991. The neurology of endemic cretinism: a study of two endemias. Brain. 114:825-841.

12. Choufoer, J.C., van Rhijn, M., and Querido, A. 1965. Endemic goiter in Western New Guinea. II. Clinical picture, incidence and pathogenesis of endemic cretinism. J. Clin. Endocrinol. Metab. 25:385-402.

13. Halpern, J.P. 1994. The neuromotor deficit in endemic cretinism and its implications for the pathogenesis of the disorder. In The damaged brain of iodine deficiency. J.B. Stanbury, editor. Cognizant Communication Co. Elmsford, New York, USA. 15-24.

14. Boyages, S.C. 1994. The damaged brain of iodine deficiency: evidence for a continuum of effect on the population at risk. In The damaged brain of iodine deficiency. J.B. Stanbury, editor. Cognizant Communication Co. Elmsford, New York, USA. 251-258.

15. Morreale de Escobar, G., Obregón, M.J., Calvo, R., Pedraza, P.E., and Escobar del Rey, F. 1997. Iodine deficiency, the hidden scourge: the rat model of human neurological cretinism. In Neuroendocrinology. C.H. Hendrich, editor. Research Signpost and Scientific Information Guild. Trivandrum, India. 55-70.

16. Pretell, E.A., et al. 1974. Iodine deficiency and the maternal-fetal relationship. In Endemic goiter and cretinism: continuing threats to world health. Volume 292. J.G. Dunn and G.A. Medeiros-Neto, editors. PAHO Scientific Publications. Washington, DC, USA. 143-155.

17. Connolly, K.J., and Pharoah, P.O.D. 1989. Iodine deficiency, maternal thyroid levels in pregnancy and developmental disorders in children. In Iodine and the brain. G.R. DeLong, J. Robbins, and P.G. Condliffe, editors. Plenum Press. New York, New York, USA. 317-331.

18. Bleichrodt, N., and Born, M. 1994. A metaanalysis of research on iodine and its relationship to cognitive development. In The damaged brain of iodine deficiency. J.B. Stanbury, editor. Cognizant Communication Co. Elmsford, New York, USA. 195-200.

19. Delange, F. 2001. Iodine deficiency as a cause of brain damage. Postgrad. Med. J. 77:217-220.

20. Chiovato, L., et al. 1994. The impact of iodine deficiency on neurological and cognitive development: the European experience. In The damaged brain in iodine deficiency. J.B. Stanbury, editor. Cognizant Communication Co. Elmsford, New York, USA. 293-298.

21. Pharoah, P.O.D., Buttfield, I.H., and Hetzel, B.S. 1971. Neurological damage to the fetus resulting from severe iodine deficiency during pregnancy. Lancet. 2:308-311.

22. Pharoah, P.O.D., Ellis, S.M., Ekins, R.P., and Williams, E.S. 1976. Maternal thyroid function, iodine deficiency and fetal development. Clin. Endocrinol. 5:159-166.

23. Xue-Yi, C., et al. 1994. Timing of vulnerability of the brain to iodine deficiency in endemic cretinism. N. Engl. J. Med. 331:1739-1744.

24. Silva, J.E., and Silva, S. 1982. Interrelationship among serum thyroxine, triiodothyronine, reverse triiodothyronine, and thyroid-stimulating hormone in iodine-deficient pregnant women and their offspring: effects of iodine supplementation. J. Clin. Endocrinol. Metab. 52:671-677.

25. Glinoer, D. 1997. The regulation of thyroid function in pregnancy: Pathways of endocrine adaptation from physiology to pathology. Endocr. Rev. 18:404-433

26. Martínez-Galán, J.R., et al. 1997. Early effects of iodine deficiency on radial glial cells of the hippocampus of the rat fetus. J. Clin. Invest. 99:2701-2709

27. Lucio, R.A., et al. 1997. The development of auditory callosal connections in normal and hypothyroid rats. Cereb. Cortex. 7:303-306.

28. Berbel, P., Ausó, E., Garcia-Velasco, J.V., Molina, M.L., and Camacho, M. 2001. Role of thyroid hormones in the maturation and organisation of rat barrel cortex. Neuroscience. 107:383-394.

29. Ausó, E., et al. 2001. Protracted expression of serotonin transporter and altered thalamocortical projections in the barrelfield of hypothyroid rats. Eur. J. Neurosci. 14:1969-1978.

30. Takahashi, T., Nowakowski, R.S., and Caviness, J.V.J. 1992. BUdR as an S-phase marker for quantitative studies of cytokinetic behaviour in the murine cerebral ventricular zone. J. Neurocytol. 21:185-197.

31. Morreale de Escobar, G., Pastor, R., Obregón, M.J., and Escobar del Rey, F. 1985. Effects of maternal hypothyroidism on the weight and thyroid hormone content of rat embryonic tissues. Endocrinology. 117:1890-1901.

32. Escobar del Rey, F., Pastor, R., Mallol, J., and Morreale de Escobar, G. 1986. Effects of maternal iodine deficiency on the L-thyroxine and 3,5,3'triiodo-L-thyronine contents of rat embryonic tissues before and after onset of fetal thyroid function. Endocrinology. 118:1259-1265.

33. Escobar del Rey, F., Mallol, J., Pastor, R., and Morreale de Escobar, G. 1987. Effects of maternal iodine deficiency on thyroid hormone economy of lactating dams and pups: maintenance of normal cerebral 3,5,3'triiodo-L-thyronine concentrations in pups during major phases of brain development. Endocrinology. 121:803-811.
34. Obregón, M.J., Ruiz de Oña, C., Calvo, R., Escobar del Rey, F., and Morreale de Escobar, G. 1991. Outer ring iodothyronine deiodinases and thyroid hormone economy: responses to iodine deficiency in the rat fetus and neonate. Endocrinology. 129:2663-2673.

35. Bayer, S.A., and Altman, J. 1991. Neocortical development. Raven Press. New York, New York, USA. 255 pp.

36. Altman, J., and Das, G.D. 1965. Autoradiographic and histological evidence of postnatal neurogenesis in rats. J. Comp. Neurol. 124:319-335.

37. Ruiz de Oña, C., Obregón, M.J., Escobar del Rey, F., and Morreale de Escobar, G. 1988. Developmental changes in rat brain 5'-deiodinase and thyroid hormones during the fetal period: the effects of fetal hypothyroidism and maternal thyroid hormones. Pediatr. Res. 24:588-594.

38. Ruiz de Oña, C., Morreale de Escobar, G., Calvo, R., Escobar del Rey, F., and Obregon, M.J. 1991. Thyroid hormones and $5^{\prime}$-deiodinase in the rat fetus late in gestation: effects of maternal hypothyroidism. Endocrinology. 128:422-432.

39. Morreale de Escobar, G., Pedraza, P.E., Escobar del Rey, F., and Obregón, M.J. 1996. Thyroidal and extrathyroidal adaptation to graded degrees of iodine deficiency: an experimental rat model for the study of neurological iodine deficiency disorders (IDD). In Thyroid and trace elements. L.E. Braverman, J. Köhrle, O. Eber, and W. Langsteger, editors. Blackwell. Vienna, Austria. 113-126.

40. Spassky, N., et al. 1998. Multiple restricted origin of oligodendrocytes. J. Neurosci. 18:8331-8343.

41. Tekki-Kessaris, N., et al. 2001. Hedgehog-dependent oligodendrocyte lineage specification in the telencephalon. Development. 128:2545-2554.

42. Valverde, F., López-Mascaraque, L., Santacana, M., and DeCarlos, J.A 1995. Persistence of early-generated neurons in the rodent subplate: assessment of cell death in the neocortex during the early postnatal period. J. Neurosci. 15:5014-5024.

43. Lavado, R., et al. 2001. Hypothyroxinemia in early pregnancy alters migration of cortical and hippocampal cells and results in neurological alterations of rat progeny. 27th Annual Meeting of the European Thyroid Association, Warsaw, Poland, 2001.J. Endocrinol. Invest. 24(Suppl. 6): 16. (Abstr.)

44. Calvo, R., Obregon, M.J., Ruiz de Oña, C., Escobar del Rey, F., and Morreale de Escobar, G. 1990. Congenital hypothyroidism, as studied in rats. Crucial role of maternal thyroxine but not of 3,5,3' -triiodothyronine in the protection of the fetal brain. J. Clin. Invest. 86:889-899.

45. Altman, J., and Bayer, S.A. 1990. Prolonged sojourn of developing pyramidal cells in the intermediate zone of the hippocampus and their setting in the stratum pyramidale. J. Comp. Neurol. 301:343-364.

46. Eriksson, P.S., et al. 1998. Neurogenesis in the adult human hippocampus. Nat. Med. 4:1313-1317.

47. van Praag, H., et al. 2002. Functional neurogenesis in the adult hippocampus. Nature. 415:1030-1034.

48. van Middlesworth, L. 1977. Audiogenic seizures in rats after severe prenatal and perinatal iodine depletion. Endocrinology. 100:242-245.

49. Rami, A., Patel, A.J., and Rabié, A. 1986. Thyroid hormone and development of the rat hippocampus: morphological alterations in granule and pyramidal cells. Neuroscience. 19:1217-1226.

50. Berbel, P., Marco, P., Cerezo, J.R., and de Felipe, J. 1996. Distribution of parvalbumin immunoreactivity in the neocortex of hypothyroid rats. Neurosci. Lett. 204:65-68.

51. Berbel, P., et al. 1993. Organization of auditory callosal connections in hypothyroid rats. Eur. J. Neurosci. 5:1465-1478.

52. Dowling, A.L.S., Iannacone, E.A., and Zoeller, R.T. 2001. Maternal hypothyroidism selectively affects the expression of neuroendocrine-specific protein A messenger ribonucleic acid in the proliferative zone of the fetal rat brain cortex. Endocrinology. 142:390-399.

53. Marín-Padilla, M. 1993. Pathogenesis of late-acquired leptomeningeal heterotopias and secondary cortical alterations: a Golgi study. In Dyslex $i$ and development. Neurobiological aspects of extra-ordinary brains. A.M. Galaburda, editor. Harvard University Press. Cambridge, Massachusetts, USA. 64-88.

54. Liu, J.L., et al. 1984. Morphologic study on cerebral cortex development in therapeutically aborted fetuses in an endemic goiter region in Guizhou. Chin. Med. J. (Engl.) 97:67-72.

55. Battaglia, G., et al. 1996. Neuronal migration disorders and epilepsy: a morphological analysis of three surgically treated patients. Epilepsy Res. 26:49-58

56. Marín-Padilla, M. 2000. Perinatal brain damage, cortical reorganization (acquired cortical dysplasia) and epilepsy. Adv. Neurol. 84:153-172.

57. Kostovic, I., and Rakic, P. 1990. Developmental history of the transient subplate zone in the visual and somatosensory cortex of the macaque monkey and human brain. J. Comp. Neurol. 297:441-470.

58. Utiger, R.D. 1999. Maternal hypothyroidism and fetal development. N. Engl.J. Med. 341:601-602.

59. Glinoer, D. 1998. The systematic screening and management of hypothyroidism and hyperthyroidism during pregnancy. Trends Endocrinol. Metab. 10:403-411. 